\title{
The relationship of global sagittal malalignment to fatty infiltration in the aging spine
}

\author{
Jonathan Charles Elysee ${ }^{1} \cdot$ Francis Lovecchio ${ }^{1} \cdot$ Renaud Lafage $^{1} \cdot$ Bryan Ang $^{1}$ (D) Alex Huang ${ }^{1} \cdot$ Mathieu Bannwarth $^{1}$. \\ Han Jo Kim ${ }^{1} \cdot$ Frank Schwab ${ }^{1}$. Virginie Lafage ${ }^{1}$
}

Received: 28 July 2020 / Revised: 1 January 2021 / Accepted: 30 January 2021 / Published online: 20 February 2021

(c) The Author(s) 2021

\begin{abstract}
Purpose To investigate associations between muscle size, fat infiltration (FI), and global sagittal alignment in patients with adult spinal deformity (ASD).

Methods Retrospective cohort study was conducted on a single-institution database of ASD patients with preoperative radiographs and CTs. Following multiplanar reconstructions of CTs, images in the plane of each vertebra were generated. The posterior vertebral musculature (PVM) was contoured on axial images at three vertebral levels (T2, T10, L3). FI was calculated by comparing Hounsfield units within muscles to the normative values of fat. Correlation analyses were conducted between demographics, alignment, and muscle characteristics.

Results 107 patients underwent preoperative spine CT (58yo, 79\%F, BMI $\left.27 \mathrm{~kg} / \mathrm{m}^{2}\right)$. Muscle data were available for 49 pts at T2, 39 pts at T10, and 81 pts at L3. Mean FI was T2 $=33 \% \pm 18, \mathrm{~T} 10=28 \% \pm 19$, L3_Erector $=39 \% \pm 19$, and L3_ Psoas $=19 \% \pm 9$. FI correlated across levels (T2 vs. T10 $r=0.698$; T10 vs L3_Erector $r=0.506$; L3_Erector vs Psoas $r=0.419)$ and with demographics; older pts had greater fat percentages $(r=0.31-0.45)$ and BMIs $(r=0.24-0.51)$. Increased FI at T2, T10, and L3 was associated with increased pelvic retroversion (PT: $r=0.25-0.43$ ), global deformity (TPA: $r=0.27-$ 0.45 ), and anterior malalignment (SVA: $r=0.23-0.41$ ). The degree of FI in the PVM increased with the severity of SRSSchwab PT and SVA modifiers.

Conclusion In ASD patients, global sagittal malalignment is related to FI of the PVM throughout the lumbar and thoracic spine, as identified through CT. Future research should investigate how FI relates to ASD pathogenesis.
\end{abstract}

Keywords Adult spinal deformity $\cdot$ Sagittal malalignment $\cdot$ CT imaging $\cdot$ Posterior vertebral musculature

\section{Introduction}

As the population ages, the prevalence of adult spinal deformity (ASD) continues to rise. While ASD is found at a rate of $32 \%$ in the general population, degenerative scoliosis may occur in up to $68 \%$ of individuals over 60 [1,2]. The disability conferred by this condition is heavily dependent on the degree of sagittal malalignment. Multiple studies have demonstrated an inverse correlation between the degree of sagittal malalignment and patient reported outcomes [3, 4]. From a mechanical standpoint, the disability attributable to the condition may be related to compensatory changes [5]

Bryan Ang

Bka2001@med.cornell.edu

1 Hospital for Special Surgery, 535 East 70th St., New York, NY 10021, USA (e.g., pelvic retroversion, hip extension, etc.) [7]. Correction of sagittal plane deformity with surgery can relieve these compensatory efforts, subsequently improving quality of life $[5,6,8]$. However, although the relationships between disability, compensation, and sagittal malalignment have been well described, the pathophysiology behind the condition is poorly understood.

While the posterior vertebral musculature (PVM) is a key component of stabilization [9], its role in the pathogenesis of degenerative spine disease is unknown. Abnormal PVM has been demonstrated in patients with a variety of conditions, including low back pain, degenerative disc disease, and lumbar radiculopathy, and patients with degenerative lumbar scoliosis; investigators have shown asymmetry between musculature on opposite sides of the curve [8-11]. Muscular changes have also been demonstrated in regional sagittal deformities [8, 10, 12-14], and relationships between fatty 
degeneration of lumbar PVM and increased global sagittal malalignment have previously been demonstrated [15]. However, given that global sagittal deformity encompasses the patient's compensatory changes in posture, it could be hypothesized that the PVM throughout the entire spine is abnormal in such cases of malalignment. Furthermore, the quality of the PVM at spinal areas other than the main zone of deformity may have important implications for postoperative outcomes [16]. Thus, the purpose of this study is to investigate associations between muscle size, FI, and global sagittal alignment in patients with ASD.

\section{Methods}

\section{Patient sample}

After approval by the institutional review board, a retrospective review of a prospectively collected, single-center database of spinal deformity patients was conducted. The database was screened for patients $>18$ years old, with a diagnosis of ASD as defined by at least one of the following parameters: Cobb angle $>20^{\circ}, \mathrm{C} 7$ sagittal vertical axis (SVA) $>50 \mathrm{~mm}$, pelvic tilt $(\mathrm{PT})>25^{\circ}$, or pelvic incidence minus lumbar lordosis mismatch (PI-LL) $>10^{\circ}$. We included patients with mainly coronal as well as primarily sagittal deformities in order to test the correlation of sagittal alignment with fatty infiltration in patients with ASD. Given this purpose, our study required a sample of ASD patients containing a wide distribution of sagittal alignments in order to test the correlation of alignment with fatty infiltration (i.e., the patients with degenerative ASD with primary coronal deformities with no sagittal malalignment served as "controls"). Exclusion criteria included any deformity secondary to trauma, inflammatory arthritis, tumors, or neuromuscular disease.

\section{Data collection}

Demographic data were collected from the electronic medical record. Lateral radiographs were analyzed using dedicated and validated software (Spineview, ENSAM, Paris). The following sagittal alignment parameters were measured: pelvic incidence (PI), PT, lumbar lordosis (LL, L1-S1), pelvic-incidence lumbar lordosis mismatch (PI-LL), T10-L2 thoracolumbar kyphosis, C7 sagittal vertical axis (SVA), and the T1 pelvic angle (TPA). Measurement of the sagittal alignment was conducted using a dedicated and validated software (Spineview) by 2 independent research technicians. Measurements were verified by a third technician in the case of measurement discrepancies.

CT scans including T2, T10, and L3 vertebrae were retrieved. Multi-planar reconstruction (MPR) of the vertebral axial plane was obtained using Osirix [17]. Using Surgimap (Nemaris Inc., New York, NY), the area of each paravertebral muscle group (PVM) was identified by manually contouring the erector spinae and multifidus (Fig. 1) $[10,18]$. This was performed at T2, T10, and L3. The psoas was also manually contoured at L3. The muscle contouring was obtained at 3 different locations: inferior to the superior end plate, in the middle of the vertebral body, and superior to the inferior end plate. Muscle analysis was conducted with Matlab R2015b (MathWorks Inc., Natick, Massachusetts) and consisted of cross-sectional area (CSA) and fat infiltration (FI). FI was expressed as the percentage of fat components (defined as the number of voxels with Hounsfield unit ranging from $-190 \mathrm{HU}$ to $\mathrm{OHU}$ ) contained within each muscle contour [12]. Specifically, axial CT slices were taken at each endplate and the middle of the vertebrae for each level and then averaged. For any outliers, the remaining two values were averaged. The percent FI from the left and right sides was then averaged to calculate the FI for that vertebral level. Similarly, to calculate the CSA for the vertebral level, the left and right CSAs were averaged. The PVM CT measurements were taken by one fellowship trained spine surgeon using Surgimap.

\section{Statistical analysis}

All statistical analyses were conducted using SPSS Version 22 (IBM, Armonk, NY). Significance was set as a type I error rate of $p<0.05$. Descriptive statistics were used to characterize demographic, radiographic, and CT-based

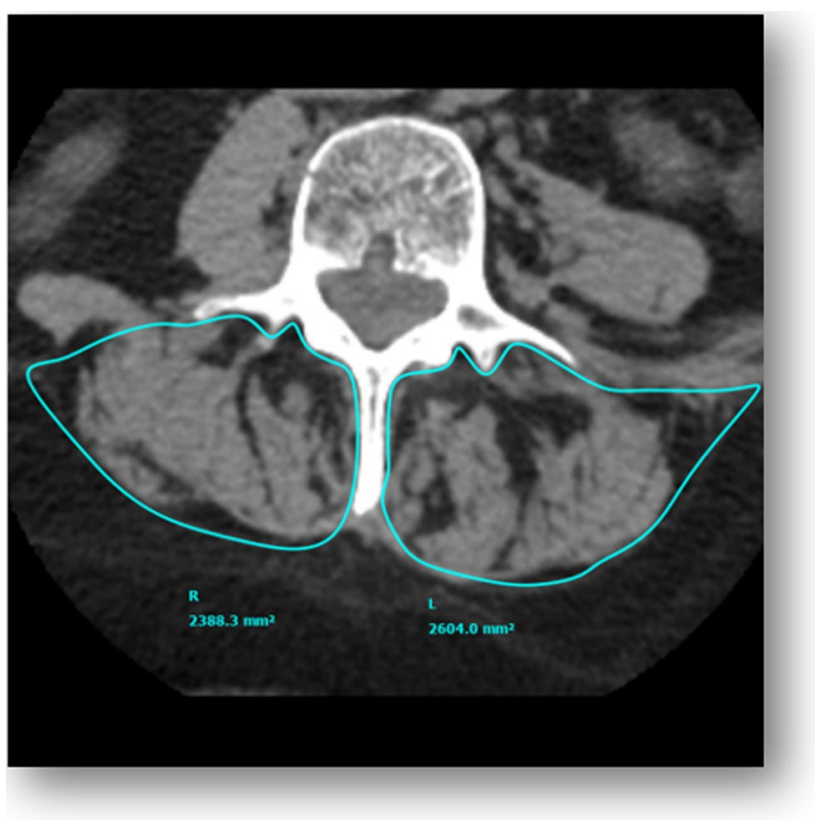

Fig. 1 Axial view showing manual contouring of the PVM 
parameters of the study sample. Non-parametric Spearman correlation analysis was utilized to determine associations between FI and age, BMI, or radiographic alignment. The cohort was classified using SRS-Schwab modifiers, and the distribution of FI was compared using Kruskal-Wallis tests [19].

\section{Results}

\section{Study sample}

A total of 107 patients met inclusion criteria for the study and had CT-scans available. The cohort included $78.7 \%$ of females, with a mean age of $57.71 \pm 17.1$ years, and a mean BMI of $26.8 \pm 6.3 \mathrm{~kg} / \mathrm{m}^{2}$. Radiographically, the population had a baseline Max Cobb mean of $43.5^{\circ}$, PI-LL mean of $16.3^{\circ}$, PT mean of $23.6^{\circ}$, and SVA mean of $66.8 \mathrm{~mm}$ (Table 1). Muscle data were available for $49(45.4 \%)$ patients at T2, $39(36.1 \%)$ patients at T10, and $81(80.6 \%)$ patients at L3. The mean FI in the erector spinae was $33.15 \%, 27.73 \%$, and $38.60 \%$, respectively, for T2, T10, and L3. The mean FI in the psoas was $18.87 \%$ (Table 1 ).

\section{Demographic and radiographic correlates with FI}

FI significantly correlated with age and BMI at all levels (Table 2), with the strongest correlations occurring at the L3 psoas and T2 erector levels ( $r=0.452$ and 0.394 ,

Table 1 Descriptive alignment parameters and fat infiltration of patient sample

\begin{tabular}{lc}
\hline & Mean \pm SD \\
\hline Radiographic parameters & \\
PI $\left(^{\circ}\right)$ & $53.7 \pm 13.9$ \\
PT $\left(^{\circ}\right)$ & $23.6 \pm 11.9$ \\
PI-LL $\left(^{\circ}\right)$ & $16.3 \pm 23.1$ \\
SVA(mm) & $66.8 \pm 82.9$ \\
Max Cobb $\left(^{\circ}\right)$ & $43.5 \pm 25.0$ \\
Muscle CSA & \\
T2_erector_CSA (mm2) & $1281.12 \pm 310.3$ \\
T10_erector_CSA (mm2) & $1781.27 \pm 540.5$ \\
L3_erector_CSA (mm2) & $3749.56 \pm 1082.5$ \\
L3_psoas_CSA (mm2) & $1867.2 \pm 523.4$ \\
Fat Infiltration percentages & \\
T2_erector_fat (\%) & $33.15 \pm 17.8$ \\
T10_erector_fat (\%) & $27.73 \pm 18.9$ \\
L3_erector_fat (\%) & $38.60 \pm 19.3$ \\
L3_psoas_fat (\%) & $18.87 \pm 9.2$ \\
\hline
\end{tabular}

$P I$ pelvic incidence, $P T$ pelvic tilt, $P I-L L$ pelvic incidence-lumbar lordosis mismatch, $C S A$ cross-sectional area, $S D$ standard deviation
Table 2 Correlation between fat infiltration and demographic parameters

respectively). Among the vertebral levels, FI and CSA were correlated (FI, T2 vs. T10: $r=0.698$; T2 vs. L3: $r=0.468$; T10 vs. L3_Erector: $r=0.506$; L3_Erector vs. Psoas $r=0.419$; CSA, T10 vs. L3: $r=0.495$; T10 vs. Psoas: $r=0.447$; T2 vs. Psoas: $r=0.500$; L3 vs. Psoas: $r=0.366$ ). Patients with higher FI at one vertebral level were more likely to have higher FI at another level, with CSA exhibiting a similar pattern.

Regarding radiographic parameters, increased FI was associated with increased pelvic retroversion, higher PI-LL, and increased anterior malalignment (Table 3). FI of the T10 and L3 erector spinae was associated with increased T10-L2 kyphosis (Table 3). Furthermore, when analyzed by CSA, a larger CSA at L3 correlated with increased LL $(r=0.37)$, lower PI-LL $(r=-0.27)$, and improved T1PA $(r=-0.22)$. At the other levels, the only other association was that a greater T2 CSA correlated with increased kyphosis at the thoracolumbar junction (T10-L2: $r=-0.31$ ). No significant association was found between Max Cobb and FI (all $p>0.1)$.

\section{Association of SRS-Schwab grade with FI}

The degree of FI in the PVM increased with the severity of SRS-Schwab sagittal modifiers for the PT and SVA modifiers, but not for PI-LL (Figs. 2, 3 and 4). Specifically, the degree of FI increased along with PT modifiers at T2, T10, and L3 (all $p<0.05$ ). For the SVA modifiers, this pattern was repeated at T2 and L3 (both $p<0.05$ ) but not T10

Table 3 Correlation between fat infiltration and radiographic parameters

\begin{tabular}{llllll}
\hline & PT & T10-L2 & TPA & SVA & Max Cobb \\
\hline T2_erector_fat & 0.352 & n.s & 0.383 & 0.348 & n.s \\
T10_erector_fat & 0.432 & -0.348 & 0.410 & 0.348 & n.s \\
L3_erector_fat & 0.367 & -0.215 & 0.407 & 0.407 & n.s \\
L3_psoas_fat & 0.247 & n.s & 0.272 & 0.453 & n.s \\
\hline
\end{tabular}

Values in the table represent Spearman rank's correlation coefficient TPA T1 pelvic angle, SVA sagittal vertical axis 


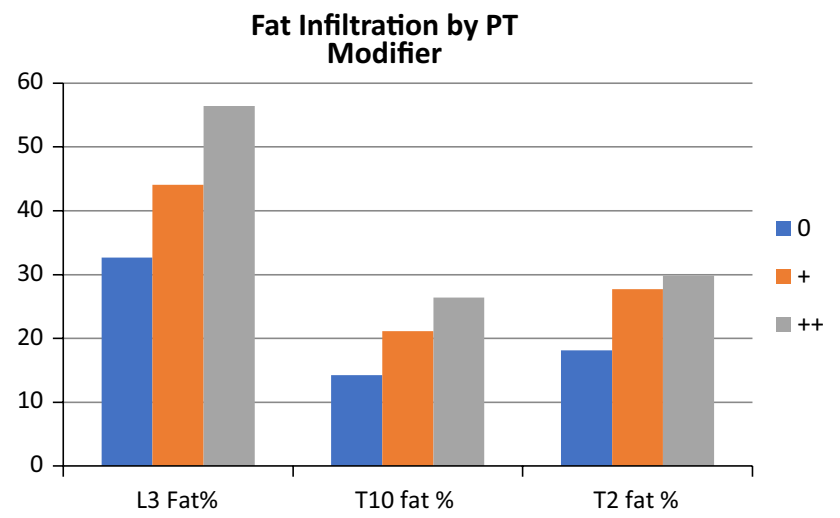

Fig. 2 Fat infiltration by SRS-Schwab PT modifier

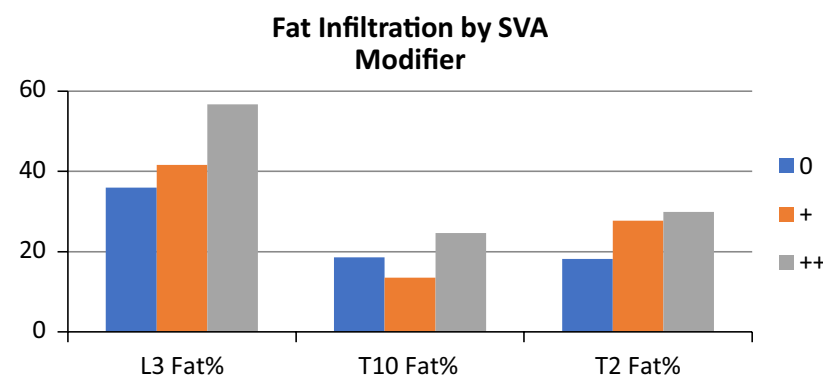

Fig. 3 Fat infiltration by SRS-Schwab SVA modifier

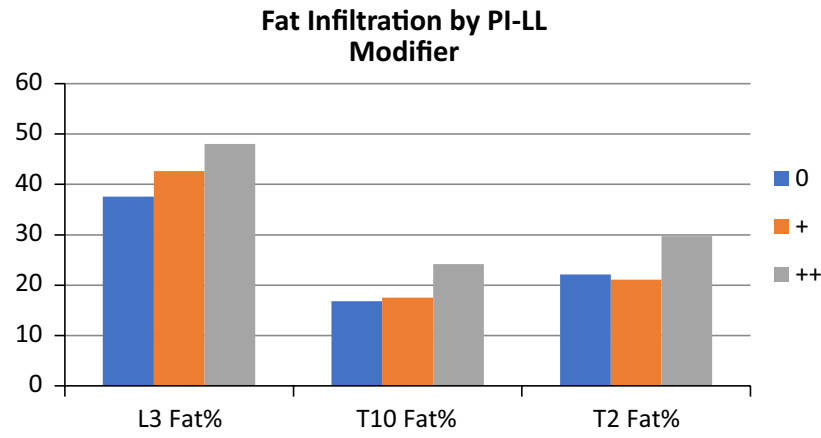

Fig. 4 Fat infiltration by SRS-Schwab PI-LL modifier

$(p=0.065)$. However, when stratified by PI-LL modifier, there were no significant differences in FI at any level (all $s>0.05)$.

\section{Discussion}

In our sample of ASD patients, we found that the degree of FI in the PVM at L3, T10, and T2 correlated with multiple parameters of global sagittal alignment. These results were supported in our secondary analysis, where increasing SRS-Schwab SVA grade correlated with increased FI. The degree of pelvic compensatory changes, represented by PT, was also correlated with FI. Relationships between regional malalignment and FI were also discovered (e.g., L3 and T10 FI and thoracolumbar kyphosis). While cross-sectional areas of the PVM were not correlated with overall sagittal alignment, local increases in CSA were associated with improved regional alignment parameters (e.g., increased L3 CSA and LL). Although causation cannot be assumed, our study supports a relationship between global sagittal malalignment and fatty degeneration of the PVM.

Older age and higher BMI were associated with FI of the PVM, helping to validate the novel method used to measure FI in this investigation, given that such associations have been corroborated by past research [12, 18]. Investigators have employed both MRI- and CT-based techniques to assess the quality of the PVM [13, 18, 20]. MRI-based modalities have quantified FI as a percentage of the PVM volume by using differences in the T2-weighted signals of fat and muscle tissue [13]. On the other hand, CT-based studies have reported the "mean density" of the PVM (averaged density over a fixed contour) expressed in Hounsfield units (HU) [18], with a lower density corresponding to a fattier PVM. Effectively, our methodology combined both approaches, using $\mathrm{HU}$ to distinguish fat cells and defining FI as a percentage of the PVM contour, and we were able to show that FI correlated with age and BMI, associations reported in multiple previous publications $[12,18]$.

Our findings are supported by previous investigations demonstrating relationships between alignment and FI of the lumbar PVM [9, 10, 15]. Lee et al. compared eleven patients with lumbar flatback (average LL $11.6^{\circ}$ ) to ten volunteers, finding a significantly higher amount of FI throughout the lumbar PVM and psoas [10], leading the authors to conclude that muscular imbalance is likely related to the pathogenesis of the condition. Similarly, Jun et al. evaluated 50 elderly patients with a wide spectrum of spinal complaints for a relationship between MRI-diagnosed L3 fatty degeneration and sagittal alignment parameters. While the diagnoses of our patient cohorts differed, they also found that that L3 fatty degeneration was associated with increased SVA, PT, and PI-LL mismatch [15]. Interestingly, the mean FI and SVA were $11.7 \pm 4.2 \%$ and $47.8 \pm 36.2 \mathrm{~mm}$ in their study, compared with a mean FI of $38.6 \pm 19.3 \%$ and SVA $66.8 \pm 82.9 \mathrm{~mm}$ in ours. Though direct comparisons cannot be made due to differences in initial diagnoses and measurement techniques, when considered together, this group of findings lends further support to an intimate role between FI of the lumbar PVM and increased sagittal malalignment.

In addition to relationships between lumbar FI and global sagittal alignment, our study adds to the literature by demonstrating how FI at T10 and T2 also correlates with increased 
SVA. The mechanical explanation for such a relationship may lie in the patient's inability to compensate through thoracic extension, as the PVM is degenerated throughout the spine. This also may explain the relationship between fatty PVM and PT, as patients with incompetent extensor musculature utilize other compensatory mechanisms, such as increasing pelvic retroversion, which relies on a different set of muscles (e.g., hamstrings). This theory is further supported by our finding that FI at L3 and T10 was associated with increased thoracolumbar junction kyphosis. The consequences of the relationship between thoracic FI of the PVM and increased SVA may also have important implications for postoperative outcomes. A univariate analysis of risk factors for proximal junctional kyphosis after thoracolumbar ASD surgery found that larger corrections of SVA and preoperative thoracolumbar FI were both associated with PJK [21]. Our study shows that these two factors are also linked. Future research on PJK, including prevention techniques, must take these relationships into account.

Importantly, our study is unable to draw any conclusions on whether fatty degeneration is responsible for sagittal deformity or vice versa. However, a reciprocal effect or "spiraling cascade" could be posited to explain the relationship between FI and sagittal deformity. Namely, excessive muscular stress or injury is known to induce abnormal muscular remodeling [20]. In the spine, animal models have shown that muscular injury can induce FI of the multifidus [22]. Thus, fatty degeneration may lead to weakness of the spine stabilizers, disabling the patient's ability to compensate and worsening the malalignment, which then increases the stress on the muscle and advances the degenerative cascade. Such a relationship has been proposed to explain fatty degeneration and kyphosis of the cervical spine [14]. The presence of radiculopathy, previously associated with FI of the PVM, may also contribute to extensor weakness through muscular denervation $[8,11]$.

We found very few associations between cross-sectional area (CSA) of the PVM and abnormal sagittal alignment parameters. In contrast to FI, CSA (or its three-dimensional equivalent, muscular volume) has not been associated with sagittal malalignment [9]. From a histologic standpoint, chronic injury to the multifidi has been shown to lead to fibrogenic gene expression and FI, but not CSA changes [22]. This pathophysiologic cascade has also been seen in other muscles intimately associated with joints, such as the rotator cuff [20]. However, we did find associations between CSA and improved sagittal alignment, such as increased LL, decreased PI-LL and lower T1PA. These findings seem to suggest that while decreased CSA is not a strong correlate with pathological conditions, increased CSA may be associated with spine health. Such associations between paravertebral muscle and bony spine health have been clinically demonstrated [23, 24]. A randomized controlled trial on the effects of back extensor exercises on preventing vertebral compression fractures in postmenopausal women found that a two-year exercise program decreased the risk of vertebral compression fractures by a factor of 2.7 [23]. While bone density is certainly a confounding factor, especially given that PVM muscle health has been related to bone density in osteoporosis research [24], the possible effect of a healthy posterior musculature "tension band" on maintaining alignment and protecting of the anterior column should not be ignored [9, 12]. On the other hand, the relationship between T2 CSA and increased thoracic kyphosis is somewhat more puzzling. One theory may be related to compensatory changes in the cervical spine-as patients increase cervical lordosis to compensate for lower thoracic kyphosis, the T2-level PVM hypertrophies [25]. Certainly, further research on the musculature about the cervicothoracic junction is warranted.

This study was not without limitations. First, due to higher prevalence of thoracic spine CTs versus MRI, we chose to use CTs to evaluate FI of the muscle, combining prior MRI- and CT-based methodologies into a novel PVM evaluation technique. However, while MRI may be superior in distinguishing fat from muscle tissue [25], the correlations reported in this study were consistent with past literature, partially validating our methodology. Furthermore, given that the same technique was utilized in every patient, the conclusions should be internally valid, especially since no conclusions were made regarding the absolute values of FI or CSA. Second, we were not able to stratify patients based on the presence of radiculopathy, which has been associated with fatty degeneration of the PVM $[8,11]$. Third, we were unable to assess the decision-making behind obtaining the preoperative CTs, possibly introducing selection bias (e.g., only patients with severe deformities may have received a preoperative $\mathrm{CT}$ scan). Fourth, we did not investigate bone density as a confounder with FI [24]. Future studies relating bone density, PVM, and malalignment are needed.

In conclusion, we found that global sagittal malalignment is related to fat infiltration of the PVM throughout the lumbar and thoracic spine in ASD patients. Our study demonstrated that CT imaging can also be easily analyzed for muscle quality. Given the relationships noted in ours and past studies [16, 23, 24], degenerative musculature may alert surgeons to patients at increased risk for mechanical postoperative complications. These findings also provide impetus for future trials looking into the effect of "pre-habilitation exercises" to strengthen core muscles and thoracic extensors to possibly reduce such complications. Lastly, while the relationship between FI and malalignment is well demonstrated, the pathogenesis of ASD remains unclear. One area of future research could involve animal models, in which FI is induced in the PVM and changes in alignment are recorded. 
Funding None.

\section{Compliance with ethical standards}

Conflict of interest None of the authors has any potential conflict of interest.

Ethical approval This study was approved by the Institutional Review Board of the Hospital for Special Surgery.

Open Access This article is licensed under a Creative Commons Attribution 4.0 International License, which permits use, sharing, adaptation, distribution and reproduction in any medium or format, as long as you give appropriate credit to the original author(s) and the source, provide a link to the Creative Commons licence, and indicate if changes were made. The images or other third party material in this article are included in the article's Creative Commons licence, unless indicated otherwise in a credit line to the material. If material is not included in the article's Creative Commons licence and your intended use is not permitted by statutory regulation or exceeds the permitted use, you will need to obtain permission directly from the copyright holder. To view a copy of this licence, visit http://creativecommons.org/licenses/by/4.0/.

\section{References}

1. Jimbo S, Kobayashi T, Aono K et al (2012) Epidemiology of degenerative lumbar scoliosis. Spine 37:1763-70

2. Passias PG, Jalai CM, Worley N et al (2017) adult spinal deformity: national trends in the presentation, treatment, and perioperative outcomes from 2003 to 2010. Spine Deformity 5:342-350

3. Yilgor C, Sogunmez N, Boissiere L et al (2017) Global alignment and proportion (gap) score: development and validation of a new method of analyzing spinopelvic alignment to predict mechanical complications after adult spinal deformity surgery. J Bone Jt Surg Am 99:1661-1672

4. Than KD, Park P, Fu KM et al (2016) Clinical and radiographic parameters associated with best versus worst clinical outcomes in minimally invasive spinal deformity surgery. J Neurosurg Spine $25: 21-25$

5. Diebo BG, Ferrero E, Lafage R et al (2015) Recruitment of compensatory mechanisms in sagittal spinal malalignment is age and regional deformity dependent: a full-standing axis analysis of key radiographical parameters. Spine 40:642-9

6. Kelly MP, Lurie JD, Yanik EL et al (2019) Operative versus nonoperative treatment for adult symptomatic lumbar scoliosis. J Bone Jt Surg Am 101:338-352

7. Solomonow M, Zhou BH, Harris M et al (1998) The ligamentomuscular stabilizing system of the spine. Spine 23:2552-62

8. Hyun S-J, Bae C-W, Lee S-H et al (2016) Fatty degeneration of the paraspinal muscle in patients with degenerative lumbar kyphosis: a new evaluation method of quantitative digital analysis using MRI and CT scan. Clin spine Surg 29:441-447

9. Ferrero E, Skalli W, Lafage V et al (2019) Relationships between radiographic parameters and spinopelvic muscles in adult spinal deformity patients. Eur Spine J. https://doi.org/10.1007/s0058 6-019-06243-3

10. Lee JC, Cha J-G, Kim Y et al (2008) Quantitative analysis of back muscle degeneration in the patients with the degenerative lumbar flat back using a digital image analysis: comparison with the normal controls. Spine 33:318-25

11. Shafaq N, Suzuki A, Matsumura A et al (2012) Asymmetric degeneration of paravertebral muscles in patients with degenerative lumbar scoliosis. Spine 37:1398-406

12. Passias PG, Segreto FA, Bortz CA et al (2018) Fatty infiltration of cervical spine extensor musculature is there a relationship with cervical sagittal balance? Clin Spine Surg 31:428-434

13. Tamai K, Grisdela P, Romanu J et al (2019) The impact of cervical spinal muscle degeneration on cervical sagittal balance and spinal degenerative disorders. Clin Spine Surg 32:E206-E213

14. Kim CY, Lee SM, Lim SA et al (2018) Impact of fat infiltration in cervical extensor muscles on cervical lordosis and neck pain: a cross-sectional study. CiOS Clin Orthop Surg 10:197-203

15. Jun HS, Kim JH, Ahn JH et al (2016) The effect of lumbar spinal muscle on spinal sagittal alignment: evaluating muscle quantity and quality. Neurosurgery 79:847-855

16. Hyun SJ, Kim YJ, Rhim SC (2016) Patients with proximal junctional kyphosis after stopping at thoracolumbar junction have lower muscularity, fatty degeneration at the thoracolumbar area. Spine J 16:1095-1101

17. Rosset A, Spadola L, Ratib O (2004) OsiriX: an open-source software for navigating in multidimensional DICOM images. J Digit Imaging 17:205-216

18. Lee SH, Park SW, Kim YB et al (2017) The fatty degeneration of lumbar paraspinal muscles on computed tomography scan according to age and disc level. Spine J 17:81-87

19. Schwab F, Ungar B, Blondel B, Buchowski J, Coe J, Deinlein D, DeWald C, Mehdian H, Shaffrey C, Tribus C, Lafage V (2012) Scoliosis Research Society-Schwab adult spinal deformity classification: a validation study. Spine 37(12):1077-82. https://doi. org/10.1097/BRS.0b013e31823e15e2

20. Hyun SJ, Kim YJ, Rhim SC (2016) Patients with proximal junctional kyphosis after stopping at thoracolumbar junction have lower muscularity, fatty degeneration at the thoracolumbar area. Spine J. https://doi.org/10.1016/j.spinee.2016.05.008

21. Hodges PW, James G, Blomster L et al (2015) Multifidus muscle changes after back injury are characterized by structural remodeling of muscle, adipose and connective tissue, but not muscle atrophy: molecular and morphological evidence. Spine 40:1057-71

22. Sinaki M, Itoi E, Wahner HW et al (2002) Stronger back muscles reduce the incidence of vertebral fractures: a prospective 10 year follow-up of postmenopausal women. Bone 30:836-841

23. Wong AK, Chandrakumar A, Whyte R et al (2019) Bone marrow and muscle fat infiltration are correlated among postmenopausal women with osteoporosis: the ambers cohort study. J Bone Miner Res. https://doi.org/10.1002/jbmr.3910

24. Hilibrand AS, Tannenbaum DA, Graziano GP et al (1995) The sagittal alignment of the cervical spine in adolescent idiopathic scoliosis. J Pediatr Orthop 15:627-632

25. Chang AE, Matory YL, Dwyer AJ et al (1987) Magnetic resonance imaging versus computed tomography in the evaluation of soft tissue tumors of the extremities. Ann Surg 205:340-348

Publisher's Note Springer Nature remains neutral with regard to jurisdictional claims in published maps and institutional affiliations. 